\title{
Volumetric Analysis is Useful for Predicting Use of Sternotomy During Retrosternal Thyroidectomy
}

\author{
George Evans 1, *, Ahad Shafi', Sanjeet Avtaar Singh', Alan Kirk ${ }^{2}$ \\ ${ }^{1}$ College of Medical, Veterinary and Life Sciences, University of Glasgow, Glasgow, UK \\ ${ }^{2}$ Department of Cardiothoracic Surgery, Golden Jubilee National Hospital, Glasgow, UK
}

\section{Email address:}

2062609E@student.gla.ac.uk (G. Evans)

${ }^{*}$ Corresponding author

\section{To cite this article:}

George Evans, Ahad Shafi, Sanjeet Avtaar Singh, Alan Kirk. Volumetric Analysis is Useful for Predicting Use of Sternotomy During Retrosternal Thyroidectomy. Advances in Surgical Sciences. Vol. 5, No. 2, 2017, pp. 15-25. doi: 10.11648/j.ass.20170502.11

Received: February 27, 2017; Accepted: March 28, 2017; Published: April 17, 2017

\begin{abstract}
Surgical removal is the recommended treatment for retrosternal goitre. This can be successfully achieved through a cervical incision in most cases, however occasionally a thoracic approach is required. Being able to predict the need for sternotomy would improve logistical efficiency, saving time and money, and allow patients to be better informed preoperatively. The aim of this study was to determine whether analysing thyroid volume on preoperative CT scans could help predict the use of sternotomy. To the best of our knowledge this is the first study investigating this. A retrospective study was conducted on 64 patients who underwent thyroidectomy for retrosternal goitre at the Golden Jubilee National Hospital, Scotland, between 2012-2016. Patient demographic information and pathological reports were obtained from databases. Preoperative CT scans were used for volumetric analysis. The total volume, the volume inferior to the level of the sternal notch (intrathoracic volume), and the anteroposterior diameter of the thoracic inlet were measured. An index of relative goitre size was calculated by dividing intrathoracic volume by thoracic inlet diameter. Mann-Whitney U tests and Fisher's exact tests were used to analyse continuous and categorical variables respectively. Goitres were successfully removed by a cervical incision alone in 55 patients, while thoracic intervention was needed in 9 cases $(13.3 \%)$. Intrathoracic volume ( $p=0.0091)$ and volumetric index $(p=0.0033)$ were significantly larger in those requiring sternotomy. Total volume was not significantly different. All other variables measured were similar in both groups. Our results suggest that assessing intrathoracic volume of goitre is useful in predicting the need for sternotomy. However, it is not able to completely rule out thoracic intervention. The volumetric index is likely to be a better predictor than volume alone.
\end{abstract}

Keywords: Retrosternal Goitre, Sternotomy, Imaging, Substernal, Thyroidectomy

\section{Introduction}

Goitre, the abnormal swelling of the thyroid gland, is usually confined to the front of the neck, and can be managed with relative ease. However, very large goitres can migrate inferiorly from the neck into the mediastinum. This phenomenon is known as retrosternal goitre. The terms substernal, mediastinal and intrathoracic are also used in the literature to describe thyroid tissue in the mediastinum.

Goitres have the tendency to extend inferiorly due to anatomical constraints in every other direction, namely the strap muscles, thyroid cartilage and the vertebral bodies. [1] Downward migration is encouraged by the traction of swallowing, negative intrathoracic pressure and gravity. [2] A short neck with short cervical trachea, and the presence of well-developed neck muscles may also facilitate downward extension of the goitre. [3]

Retrosternal goitre is most often diagnosed in the $6^{\text {th }}$ decade of life, and is around 4 times more likely in females. [4] The literature about incidence of malignancy in retrosternal goitre is conflicting, but has been stated as between 3 and 21\%. [5] Certain studies show a higher incidence of malignancy in retrosternal goitre compared to cervical goitre, [6] while other sources claim the incidence is no higher. [7]

There is no clear consensus on what exactly characterises retrosternal goitre, and a myriad of different definitions can 
be found in the literature. These range from simply any goitre which extends below the plane of the thoracic inlet, to more stringent criteria, such as having greater than $50 \%$ of the total volume found in the mediastinum, or extending at least $3 \mathrm{~cm}$ below the suprasternal notch. The incidence of retrosternal goitre varies substantially across the literature, mainly due to these differences in definition. [8] One study showed that the incidence of retrosternal goitre in their group of patients could vary from $2.8 \%$ to $48 \%$, if using the most and least restrictive definitions respectively. [8]

Retrosternal goitres can be classified as primary or secondary. Secondary retrosternal goitres are those which extend inferiorly from the original position in the neck. Primary intrathoracic goitres are formed from ectopic thyroid tissue located in the mediastinum. They are not connected to the cervical thyroid and receive their blood supply from mediastinal vessels, as opposed to branches of the inferior thyroid artery. [9] Primary intrathoracic goitres are uncommon, making up less than $1 \%$ of retrosternal goitres. [10]

Retrosternal goitres are usually slow-growing and are often asymptomatic for years. A multicentre study showed that $27 \%$ of retrosternal goitre patients were asymptomatic when admitted for surgery. [6] Large retrosternal goitres can cause symptoms due to compression of surrounding structures, especially at the thoracic inlet region where any large mass between the rigid manubrium and vertebrae is likely to compress other structures. [5] Over 50\% of retrosternal goitre patients are affected by respiratory problems occurring due to compression of the trachea, including dyspnoea, choking and hoarseness. Compression of the oesophagus causes around a third of patients to experience dysphagia. [11] Less frequently it can affect vascular or neural structures, manifesting as problems such as superior vena cava syndrome in $5-9 \%$ of patients [11] or Horner's syndrome. [10] There is usually an uncomfortable mass in the neck, however up to $30 \%$ of retrosternal goitres are barely palpable. [7] In addition, patients may experience the effects of thyroid dysfunction which can accompany goitre.

Medical treatment is largely futile and only temporary, producing only a partial reduction in the size of goitre. Surgical removal of the goitre, known as thyroidectomy, offers a definitive treatment. It is generally agreed that surgery should be performed for retrosternal goitre, even in the absence of symptoms. This is because a sudden enlargement of the goitre can be life-threatening, and because malignancy could be missed. Performing fine-needle aspiration biopsy on retrosternal goitre is both difficult and dangerous, with risk of mediastinal bleeding preventing reliable sampling of the intrathoracic portion. [3]

Most retrosternal thyroidectomies are performed using a cervical approach. This involves making a horizontal incision in the neck, usually measuring 3 or 4 inches. The strap muscles are separated, and thyroid blood vessels are identified and ligated. Care must be taken to identify the recurrent laryngeal nerve, and an effort should be made to locate and preserve the parathyroid glands. The gland is delivered up out of the neck using gentle upward traction over the superior pole. [12] If only one thyroid lobe is affected, while the other is normal, then a lobectomy may be performed instead of a total thyroidectomy.

When the goitre cannot be removed using a cervical approach, an extracervical approach is required. Success rates of greater than $99 \%$ have been reported using the cervical approach, [12] while other studies have shown that up to $30 \%$ of patients require thoracic intervention. [13] Again, the incidence of extra-cervical approach being required is affected greatly by which definition of retrosternal goitre is used. Most studies state an incidence of around 10\% or lower. In most cases when the goitre cannot be removed through the cervical incision, the surgeons opt to perform a full median sternotomy. Other thoracic approaches may be utilised such as manubriotomy or posterolateral thoracotomy, especially if the goitre extends into the posterior mediastinum.

Although the goitre is more likely to be successfully removed if sternotomy is utilised, a cervical approach is the recommended method because of preferable morbidity and mortality outcomes. The most common complications include bleeding, recurrent laryngeal nerve injury, hoarseness and hypoparathyroidism. These are uncommon thanks to good modern surgical technique. Tracheomalacia is another complication that has been well-described in the literature, however it is very rare. One large study study compared morbidity between the two surgical approaches for retrosternal goitre, and did not find any significant differences in complications. [6] Unplanned postoperative intubations and transfusions are required more often in patients undergoing a transthoracic approach. [14] These results may be affected by selection bias, as patients requiring sternotomy may have larger goitres which could be the reason for the increased complications, and the surgeon may be more willing to accept greater blood loss during sternotomy. Sternotomy is associated with pulmonary complications such as pneumothorax, atelectasis and pneumonia. [15] Sternotomy also leaves a large scar, causes greater pain and has greater chance of wound infection. The cervical approach is also preferable as the surgery is quicker and it is associated with shorter postoperative hospital stay. [6]

The consensus is that the best approach is to always aim to perform the thyroidectomy via the cervical approach, and the decision to use sternotomy should only be made intraoperatively once every effort has been made to safely remove the goitre through the neck. Thyroidectomy is usually performed by head and neck, ENT, endocrine or other similar surgical specialties. However, it is thoracic surgeons who perform sternotomy and other thoracic procedures. This means that both surgical teams must be present at the operation, even though the services of the thoracic surgeon are only required in a minority of cases.

Getting both surgical teams together at the same time can take a significant amount of planning. As was the case in this 
study, the surgical teams can be located at separate hospitals. If this is the case it involves working in an unfamiliar environment, which could affect the performance of the surgery. The thoracic surgeon's time could be better spent on other patients. Patient movement can add to the financial expense.

If there was a way of knowing preoperatively whether sternotomy is needed, it could improve planning and enable better use of resources. It would save money, improve safety and make the process more efficient logistically. Having a better understanding of what the operation will involve, allows more accurate information to be discussed with the patient preoperatively and aids in their decision to consent.

Currently there is no definitive way of determining whether sternotomy will be needed. Studies have identified various factors associated with the need for sternotomy. Many studies have investigated the anatomical position of the goitre, with inferior extension being the most prevalent characteristic. Various studies have claimed different amounts of inferior extension are significant, with the following landmarks being identified: inferior to the aortic arch, [5, 16-19] or below the carina. [20-22] Evidence consistently claims that posterior mediastinal goitres are more likely to require thoracic intervention than anteriorly located goitres [16, 17, 21, 22]. Mercante et al. 2011, developed a classification system based on anatomical position to predict extracervical surgery. [23]

Sternotomy is required for most primary intrathoracic goitres. $[12,21]$ Thyroid tissue density determined from CT scans was identified as a strong predictor of sternotomy by one study. [22] Longer history of retrosternal goitre before surgery, [16] fibrosis or scarring from prior radiation or surgery [11] and right sided goitre [17] have been stated to be risk factors for sternotomy. Many believe malignancy is a risk factor for sternotomy. [19] Recently a large study showed no statistical difference in malignancy between surgical approaches. [14] Some studies where it was not shown to be a risk factor, still state that they think it does contribute towards need for sternotomy due to invasion of surrounding structures and any nodal infiltration. [10] It has been highlighted that the rate of sternotomy is likely to be lower if performed by experienced surgeons in high volume centres.

Size and shape of goitre influence risk of sternotomy. 'Conical', 'iceberg-' and 'dumbbell-shaped' are associated with sternotomy. These shapes are problematic as the thoracic inlet acts as a ring of constriction. Essentially when the goitre is wider than the thoracic inlet, sternotomy is more likely to be required [19, 20]. Estimated volume has been suggested as a factor, and the weight of the excised glands has been shown to be significant, [10] however nobody has quantitatively measured volume.

CT scans are the imaging modality of choice and should be done on all patients with retrosternal goitre. [2, 11, 21] In this retrospective study, a segmentation tool was used to measure goitre volume on CT scans, to investigate the relationship between volume and surgical approach. By performing volumetric analysis and reviewing other clinical data we aimed to determine whether goitre volume was related to risk of sternotomy. To the best of our knowledge this is the first time this has been done.

\section{Materials and Methods}

A retrospective study of all thyroidectomies performed at the Golden Jubilee National Hospital (GJNH), a regional cardiothoracic centre in Scotland, was undertaken. 64 patients met our criteria and were included for volumetric analysis.

Demographic information including date of birth and gender was collected for each patient using a patient database. Referral and discharge letters, and operation notes were reviewed to gather information on the named procedure, operation date, surgical approach, surgeons involved, complications and any additional information that was thought to be relevant. Pathological information was obtained from pathology reports.

Volumetric analysis was performed using the most recent preoperative CT scans available. The date of the scan was recorded and the time between scan and operation calculated. CT scans were obtained using machines in a variety of hospitals. There may have been slight variations in CT protocols. Scans were viewed using CARESTREAM Vue PACS Version 11.4.1.1102 (Carestream Health Inc (Onex Corporation, Canada)). The in-built lesion management tools were used to perform semi-automated segmentation of goitres. The process of segmentation involved tracing around the goitre on an axial slice of the CT scan using the livewire segmentation tool (Fig. 1a). Three methods of tracing were available and could be used together to save time and enable accurate segmentation: 1) moving mouse around goitre allowing software to automatically identify lesion boundary; 2) clicking at key points to assist software in identifying boundaries; 3) holding down click allowed manual line drawing without any input from the software. Once delineated in two or more CT slices the software automatically segmented the goitre in between these slices. This software is mostly used to measure lesions in the lung and liver, and boundaries of goitres tend not to be as clear as these lesions. This along with the multinodular nature of most goitres, meant the software was not always accurate at automatically circumscribing the goitre. Consequently, a lot of manual adjustment was often required to accurately segment the goitre. As the thyroid has two lobes, there were often two or more separate portions that had to be combined using the merge tool. The software automatically calculated the volume of segmented lesions. Studies, including one examining complex brain lesions, have confirmed the accuracy of this software. [24, 25]

Total volume of goitre was recorded. The intrathoracic goitre volume was also determined. Using the sagittal view (Fig. 1b), a horizontal line was drawn from the suprasternal notch using the crop tool. This removed the smaller portion of the segmented goitre. If the volume inferior to the plane was removed (in cases where the cervical portion was larger), 
the volume of the remaining portion was subtracted from the total volume to determine the intrathoracic volume. Using another plane such as the diagonal plane of the first ribs was considered for separating the intrathoracic portion of the goitre. This was difficult to execute so the horizontal method was selected. Thoracic inlet diameter was measured by finding the shortest distance from the sternum to the vertebral column (Fig. 1b). This measurement was also made using a sagittal view, while simultaneously checking an axial view to confirm correct alignment. A volumetric index was calculated by the following calculation: intrathoracic volume $\left(\mathrm{mm}^{3}\right)$ divided by thoracic inlet diameter $(\mathrm{mm})$, and then divided by 1000 . We created this volumetric index as a way of quantifying the goitre size relative to the size of the thoracic inlet.

\section{Volumetric Index $=$ Intrathoracic volume $\div$ Thoracic inlet diameter $\div 1000$}
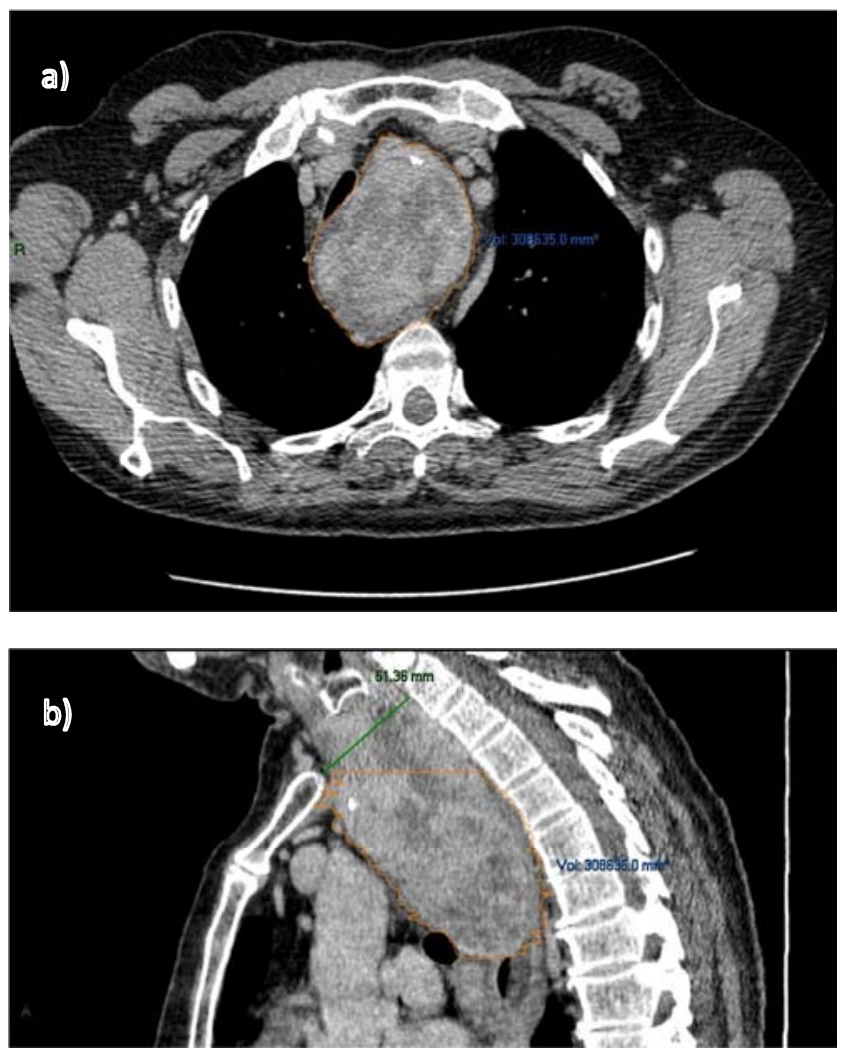

Figure 1. Segmented retrosternal goitre on CT scan in CARESTREAM Vue PACS.

a. Axial view of retrosternal goitre. Orange line demonstrates segmentation of the goitre which is located in the mediastinum. The volume is shown in blue text. Compression of the trachea can be seen.

b. Sagittal view, with intrathoracic portion of goitre surrounded by orange line. Green line demonstrates thoracic inlet diameter, defined as the shortest distance from the sternum to the vertebrae.

The definition of retrosternal goitre chosen for this study was, any goitre with radiological extension below the plane of the thoracic inlet. As [21] alludes, this plane is intuitive, fixed, and does not rely of judgement of proportionality. 10 patients were excluded from volumetric analysis for the following reasons: problems using lesion management software (4 patients), no CT scan available (2 patients), no extension below thoracic inlet radiologically (3 patients), planned thoracotomy ( 1 patient). The patient who underwent a right posterolateral thoracotomy was excluded because this was the planned approach, and it was not clear from letters whether he had undergone previous thyroid surgery via a cervical approach.

Patients were placed in 2 groups: those who needed sternotomy, and those requiring a cervical incision only. Fisher's exact test was used to compare categorical variables between the two groups. An independent two-sample t-test was used to compare age between the groups. Total volume, intrathoracic volume, thoracic inlet diameter and volumetric index were analysed using the Mann-Whitney $U$ test. Anderson-Darling Normality tests were utilised to check normality of distributions. $\mathrm{P}$ values of $<0.05$ were considered significant for all tests. All statistical analysis was performed using Minitab ${ }^{\circledR} 17$ Statistical Software (Minitab Inc. 2010).

\section{Results}

Of the 74 patients who had thyroidectomies at the GJNH, 64 were included in this study. Patients were excluded because they did not meet our criteria or scans were not available. The cervical approach was adequate in 55 cases $(86 \%)$, with a thoracic approach being required in 9 cases (14\%). A median sternotomy was used in 7 cases, while manubriotomy and parasternal mediastinotomy were both used once.

36 total thyroidectomies and 28 hemithyroidectomies were performed, however, in each case the entire retrosternal portion was removed. 4 cases were a completion or re-do thyroidectomy, with one of these requiring sternotomy. At least 15 different surgeons were involved in performing thyroidectomies in this study. One patient's CT scan did not show part of the cervical portion of the goitre and thoracic inlet diameter was not able to be calculated. This patient was excluded from analysis of total volume and volumetric index, but included for intrathoracic volume analysis. Exclusion of this result did not alter the significance of the result of the Mann-Whitney $U$ test for intrathoracic volume.

51 of the patients $(80 \%)$ were female. Patient age (at time of surgery) ranged from 38 to 84, with a mean age of 64 . Malignancy (excluding microcarcinomas) was present in 8 cases (12.5\%). Malignancy was of the following types: 2 patients had follicular carcinoma, 2 patients had anaplastic carcinoma, 2 patients had parathyroid carcinomas and there were single cases of papillary and medullary carcinomas. 53 patients had multinodular goitre, with a few patients having follicular adenomas and one case of an incidental microcarcinoma. Complications were encountered in 13 patients $(20 \%)$, including 3 cases of bleeding or high output wound drains, temporary hoarseness in 3 patients, 4 cases of hypocalcaemia, 2 cases of hypertension, a chyle leak, and postsurgical atelectasis. The patient with atelectasis had undergone sternotomy. The low numbers in this study did not allow for analysis of complication rates, but enabled comparison of any 
obvious differences between the groups (Table 1). There were no deaths associated with the procedure.

Table 1. Demographic, pathologic and complications data for patients undergoing retrosternal thyroidectomy. Information was compared between patients whose operation was successful by neck incision alone, and those who required sternotomy. The mean ages and standard error are shown. Statistical analysis was performed using a two-sample t-test for age, and Fisher's exact test for categorical variables. P-values are shown, and there were no significant differences between the two groups. All cases of hoarseness were temporary.

\begin{tabular}{llll}
\hline Details & Sternotomy $(\mathbf{n = 9})$ & Cervical $(\mathbf{n = 5 5})$ & P-value \\
\hline Demographics & & & \\
Age & $68.7 \pm 2.4$ & $64.1 \pm 1.6$ & 0.2653 \\
Gender (M: F) & $2: 7$ & $11: 44$ & 1.000 \\
Pathology & & & \\
Malignant & 1 & 7 & 1.000 \\
Complications & & & \\
Hoarseness & 0 & 3 & 1.000 \\
Hypocalcaemia & 1 & 3 & 0.4632 \\
Bleeding & 0 & 3 & 1.000 \\
Atelectasis & 1 & 0 & 0.1406 \\
\hline
\end{tabular}

Demographics were compared between sternotomy and cervical groups to ensure this was not influencing results (Table 1). Both age and gender were similar between the two groups. No difference in malignancy rates was found between the groups. Rates of common complications did not differ between the surgical approaches (Table 1).

A trend existed between larger total goitre volume and the requirement for thoracic intervention. However, total volume was not significantly different between the two groups. Descriptive statistics for the distribution of total volumes can be found in the appendix.

Intrathoracic volumes in the cervical group ranged from $2938 \mathrm{~mm}^{3}$ to $272,141 \mathrm{~mm}^{3}$ with a median of $48,732 \mathrm{~mm}^{3}$. While for the sternotomy group intrathoracic volume ranged from $33,206 \mathrm{~mm}^{3}$ to $409,155 \mathrm{~mm}^{3}$, with a median of $167,552 \mathrm{~mm}^{3}$ (Fig. 2). The mean intrathoracic volumes were $88,063 \mathrm{~mm}^{3}$ and $196,471 \mathrm{~mm}^{3}$ respectively. Intrathoracic volumes were not normally distributed for the cervical group. Histograms can be found as an appendix. Using the MannWhitney $U$ test, intrathoracic volume was found to be significantly larger in the sternotomy group giving a $\mathrm{p}$ value of 0.0091 .

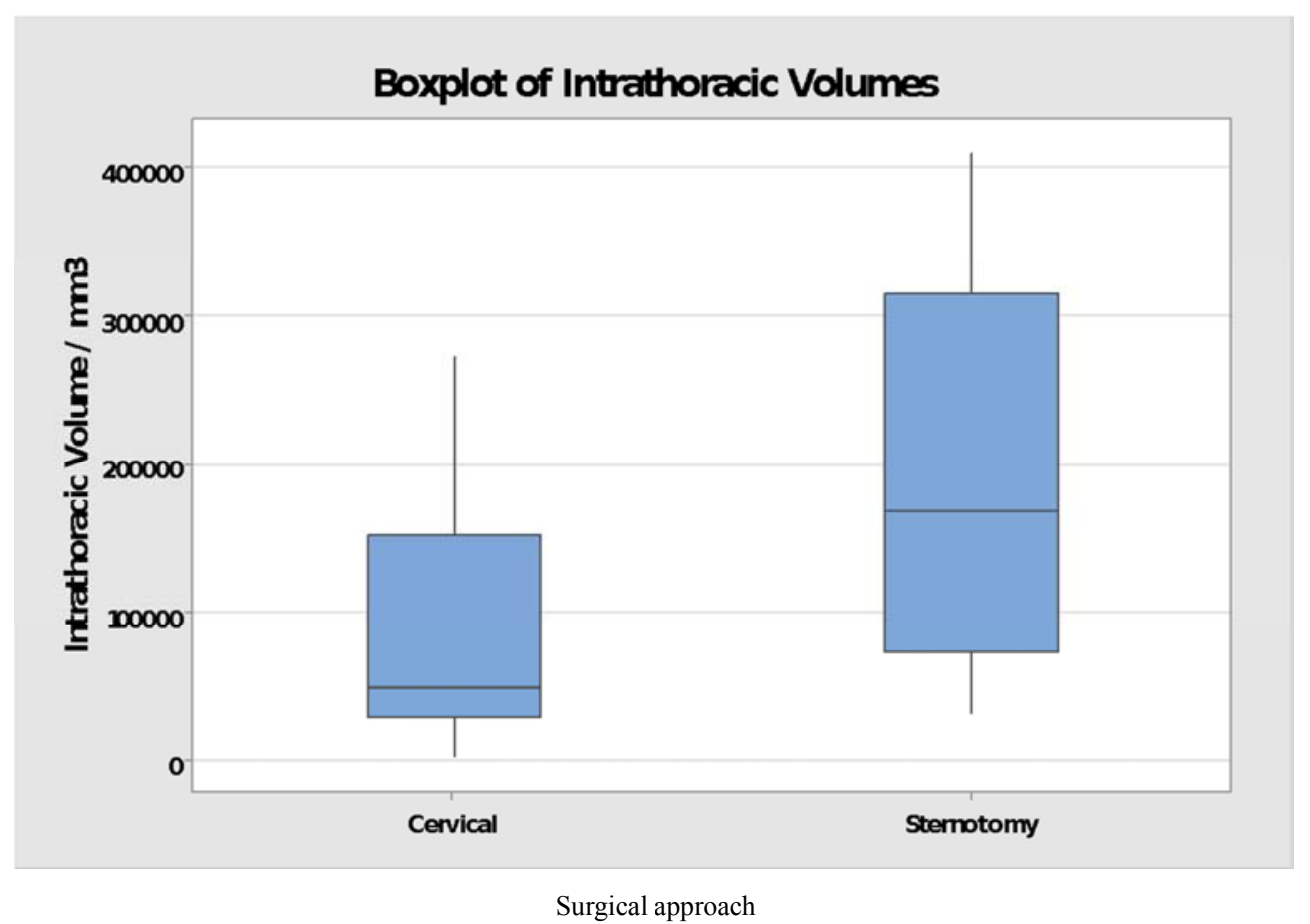

Figure 2. Boxplot of Intrathoracic goitre volume for retrosternal thyroidectomy patients who were operated on by the cervical approach and by sternotomy. The median, quartiles, and minimum and maximum values are shown for each group. Intrathoracic volume was the portion of goitre located inferior to the level of the suprasternal notch. Volumes for those who required sternotomy were significantly larger p=0.0091. 55 patients needed a cervical incision only, and 9 patients required sternotomy.

A volumetric index of volume divided by thoracic inlet diameter was calculated. The average thoracic inlet diameter was similar between the two groups. Volumetric index was significantly larger in the sternotomy group than the cervical group. The Mann-Whitney $U$ test gave a p-value of 0.0033 . Volumetric indexes ranged from 0.758 to 7.174 in the sternotomy group, and from 0.053 to 6.545 in the cervical group (Fig. 3). The mean indexes were 3.793 and 0.915 respectively. The minimum value for the sternotomy group is close to the median value of the cervical group. The star seen in figure 3 is a statistical outlier, that was included in analysis. In patients receiving sternotomy, only two had a volumetric index score of less than 2 (Fig. 4). In the surgical notes, surgeons had stated that one of these patients had an unusually short neck, and the other was morbidly obese. The sternotomy patient with an index of 2.047 had a difficult intubation, but the reason behind this was unclear. The next smallest index of a sternotomy patient excluding these three is 3.425 . Descriptive statistics about 
volumes can be found in an appendix.

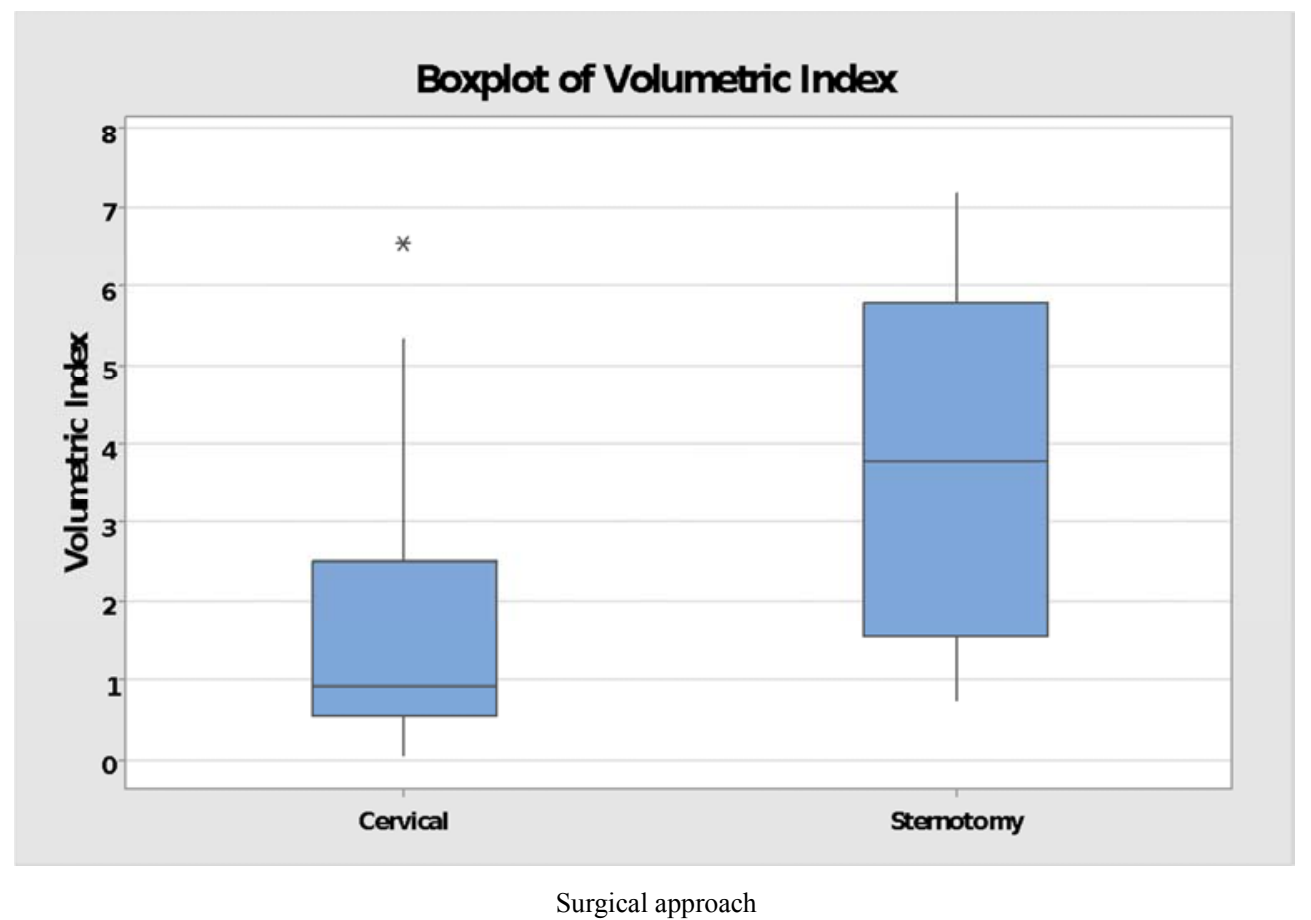

Figure 3. Boxplot of volumetric indexes for retrosternal thyroidectomy patients who did or did not require sternotomy. Volumetric Index $=$ Intrathoracic volume $\div$ Thoracic inlet diameter $\div 1000$. The star on the boxplot of volumetric indexes for the cervical group shows a statistical outlier. Median, quartiles, and minimum and maximum values are indicated for patients in both groups. Data from 9 patients requiring sternotomy, and 54 patients needing cervical access alone, is included. The volumetric indexes for patients requiring sternotomy were significantly larger $(p=0.0033)$ than for patients who did not need sternotomy.

One of the most commonly used definitions of retrosternal goitre is 'at least $50 \%$ of goitre is retrosternal' (Katlic's definition). [26] Ríos et al. 2010, claim that this is the best definition for predicting sternotomy. [8] Further analysis of data was performed using only those patients who met Katlic's definition of retrosternal goitre. Many patients in the cervical group had very small intrathoracic volume. Katlic's definition removed most of these very small values. 26 patients had retrosternal goitre per Katlic's definition, 19 of which were removed through a cervical incision alone, and 7 of which required sternotomy. 2 patients who required sternotomy did not meet this definition of retrosternal goitre. There was a trend towards larger intrathoracic volumes in the sternotomy group, with the Mann-Whitney U test giving a pvalue of 0.1186 . There were significantly larger volumetric indexes in those who needed a sternotomy (Fig. 5), with a Mann-Whitney $U$ test generating a p-value of 0.0494 . This analysis shows that even when smaller goitres are excluded, sternotomy is more likely to be required for the largest goitres. This reinforces the suggestion that volume is a significant factor in determining sternotomy requirement. Volumetric index was significant while volume alone was not, indicating that index could be a greater tool than volume for predicting sternotomy.

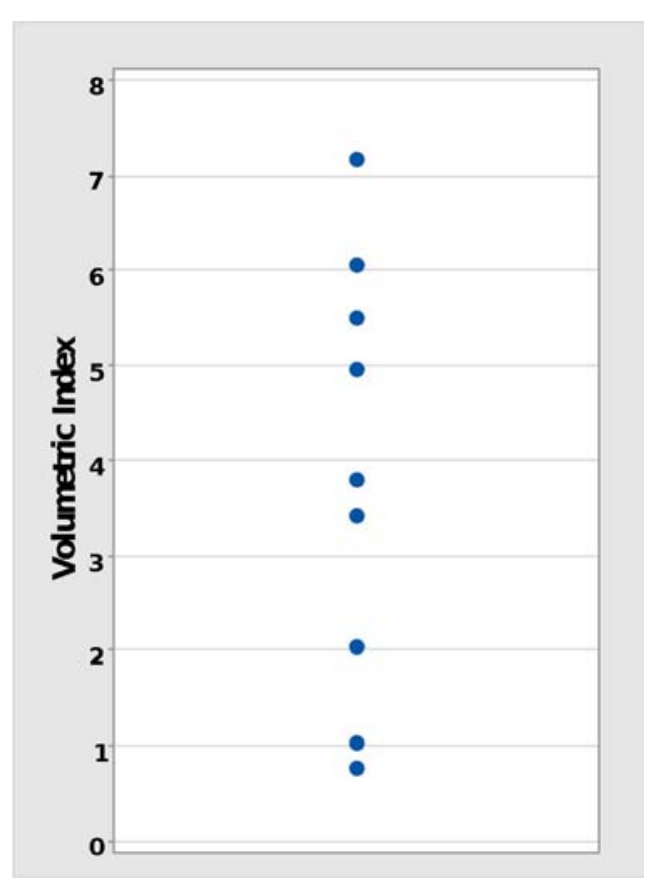

Figure 4. Individual value plot of volumetric indexes in patients requiring sternotomy for retrosternal thyroidectomy. Each dot indicates the volumetric index of an individual sternotomy patients. In total, 9 patients required sternotomy.

Volumetric Index $=$

Intrathoracic volume $\div$ Thoracic inlet diameter $\div 1000$. 


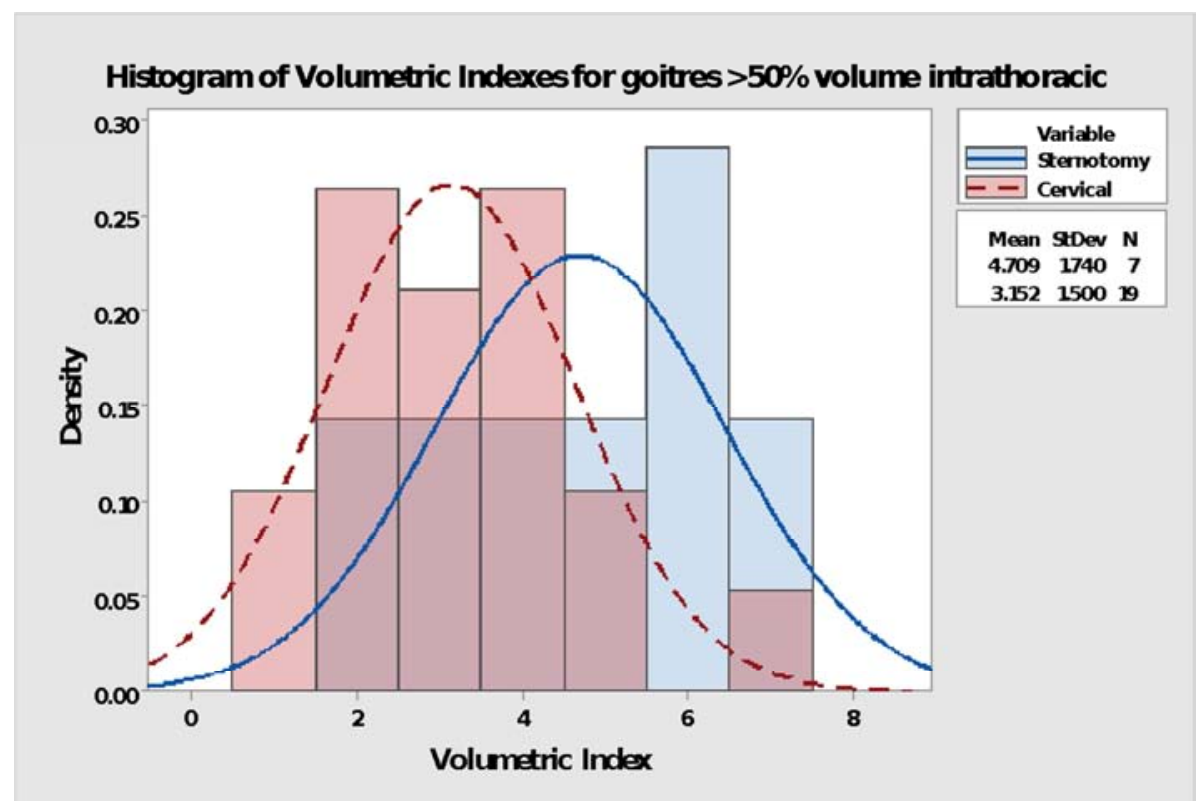

Figure 5. Histogram showing distribution of volumetric indexes for patients undergoing retrosternal thyroidectomy. It includes only patients who met Katlic's definition of retrosternal goitre: $>50 \%$ of goitre volume is retrosternal (Katlic et al. 1985). The blue bars indicate patients who required sternotomy to remove their retrosternal goitre. The red bars indicate patients who had their retrosternal goitre removed using only a cervical incision. The graph shows the relative distribution of each patient group's volumetric indexes. 7 patients needed sternotomy, and 19 patients did not. It is important to note that the y axis shows the density or proportion of each group for each value, not the number of patients. Volumetric Index $=$ Intrathoracic volume $\div$ Thoracic inlet depth $\div$ 1000. Volumetric analysis is a continuous variable. Patients in the sternotomy group have significantly larger volumetric indexes ( $p=0.0494$ ). Mean and standard deviation for each group in displayed on the right-hand side.

\section{Discussion}

This study showed that the average intrathoracic goitre volume is larger in patients who require thoracic intervention to remove their retrosternal goitre, compared with patients whose retrosternal goitre can be successfully removed using a cervical approach alone. An index of goitre volume over thoracic inlet diameter is also significantly larger in patients who require sternotomy. This means larger intrathoracic volume does contribute towards the need for sternotomy.

Total volume was not significantly different between the two groups, in agreement with a study that showed that weight was not significant because of large cervical components. [5] We expected a greater difference for intrathoracic volume as many goitres had substantial volume in the cervical region, which would not be relevant to needing to open the thoracic cavity. Hypothetically, a goitre may be removed more easily in a person with a large thoracic inlet than the same sized goitre in a person with a smaller thoracic inlet. The volumetric index was used for this reason. Average thoracic inlet diameter was similar between groups, showing that small thoracic inlet alone is not a significant risk factor for sternotomy.

Although other studies have claimed that goitre size is significant in predicting the need for sternotomy, [4] this is the first study which quantitatively analysed how volume compares between patients who do or do not need sternotomy. Studies have measured other aspects of size, notably inferior extension, while maximal width compared with thoracic inlet size has also been mentioned. [19] Volumetric index should be correlated with these other measures, and should give a good overall measure of size relevant to surgical difficulty. Volumetric index was also significant when using a more restrictive definition of retrosternal goitre, while intrathoracic volume alone was not. This suggests a volumetric index of volume relative to thoracic inlet is more valuable for predicting sternotomy, than intrathoracic volume alone.

Demographic, pathology and complication data in this study is consistent with the literature. [5, 7] However, [12] had a much higher rate of hypocalcaemia, possibly because they performed total thyroidectomy in almost all cases, whereas hemithyroidectomies were commonly performed in this study. In agreement with figures from [14], no difference in malignancy rates were found between the groups. While [10] had similar results, they still consider malignancy as a high risk of sternotomy.

Preoperatively measuring goitre volume on CT scans could be useful for planning operations. This suggestion is practical, as it is recommended that CT scans should be performed on all retrosternal goitre patients. [11] If the volume is large, it would prompt assistance of a thoracic surgeon to be arranged, facilitating a greater chance of safe and successful surgery. If a small volume was measured, it would encourage efficient use of resources, saving time and money.

Volume alone cannot predict sternotomy, but instead is one of many factors which increases the probability of extracervical approaches being required. The distribution of 
values in this study overlapped between the two groups, reinforcing that other factors, such as posterior mediastinal involvement [22] are important. Although the outlier in this study was a large goitre successfully removed cervically, it highlights that outliers are possible. This raises the possibility that some scenarios cannot be predicted, and some surgeons may take the precaution of thoracic help regardless of predictive methods, in the interest of patient safety and completing the operation first time around. As shown in this study, patients with a volumetric index less than 2 only needed a sternotomy in 2 cases. One of these patients had an unusually short neck, and the other was morbidly obese. These characteristics made the surgery more difficult and undoubtedly contributed towards the need for sternotomy. Perhaps a value of 2 for volumetric index could be a potential cut off point, below which thoracic help shouldn't be requested unless the patient has another risk factor for sternotomy.

The tools used for measuring volume are built in to Carestream Vue PACS, which is used across Scotland and other healthcare services. This is the first documented use of this software for measuring thyroid volume, however it has been shown to be accurate for other complex masses. [24] Automatic segmentation of goitres using this software was not accurate, so a lot of user input was required to segment with reasonable accuracy. After using for a short time, the software was simple to operate and volume could be determined within a few minutes. There were a few problems with the software which made segmentation difficult or unachievable. 4 patients were excluded because the volumetric analysis software did not work on their CT scans. This appeared to be because of a fault with the scans as the slices were not correctly aligned. It is not clear whether this could have been fixed. There has been increasing development of tools for measuring thyroid volume, which are quick and accurate. [27, 28] Improved segmentation will help clinicians and future studies analyse goitre volume.

Volumetric analysis provides a quantitative measure of size, which in previous studies was estimated and subjective. The thyroid has a complex three-dimensional structure, meaning that width may not be relevant and the most relevant dimension may be hard to measure on CT. Volume could be a more reliable measure for this reason. Volumetric analysis is time consuming compared with measuring width or estimating size. Having a quantitative measure is better than estimating as the information can be used in research and guidelines can be created in relation to numbers.

Limitations of this study included the sample size. It limits the power of any conclusions drawn. This was a retrospective study, relying on accurate record keeping of others, and occasionally information was not completely clear. Other factors could not be controlled. Some patients were excluded due to unavailability of CT scans or the software not working. None of these patients required sternotomy, so their omission is unlikely to significantly affect results. There may have been slight differences in CT protocols which could affect the results for intrathoracic volume. Neck extension can shift the goitre superiorly by $10 \mathrm{~mm}$ at the level of the manubrium, but does not change position in relation to mediastinal structures [17]. Thus, minor changes in results may occur if there were inconsistencies in CT protocols. The shape of a patient may have affected the horizontal line used to separate the intrathoracic portion of the goitre. This is because a more prominent spinal kyphosis could change the angle of the thoracic inlet when lying flat on the CT scanner. Any variation here is likely to be minimal, and the approach used for identifying the boundary was the most practical. There was a mean of 168 days between CT scan and operation. During this time, there was potential for growth, and thus reported volumes may be inaccurate, however, goitres are usually slow-growing. [10] The number of different surgeons involved in this study could be seen as a limitation, however it could be argued that it makes results more generalizable.

Now that volume is proven as a significant factor, future studies may wish to include volumetric analysis, and build up more evidence. Larger studies may drive volumetric analysis to be part of preoperative assessment. Large studies will enable regression analysis, which can determine odds ratios, and specific volumes that present a greater risk of sternotomy. Once enough evidence is compiled, development of a standardised scoring tool which predicts sternotomy using all the different risk factors, could be possible. This would be the ideal way of predicting sternotomy, and could have the potential to increase health service efficiency and surgical success. Very few large studies about retrosternal goitre exist. The low incidence of retrosternal goitre makes large study populations difficult to obtain. Performing a large study would take years, during which time surgical practice could change. Multicentre studies are a quicker option, but have limitations. Currently, it is the surgeons personal experience and opinion that may be the best tool for predicting sternotomy and hence arranging cooperation with thoracic surgeons. Inferior extension, posterior mediastinal position and ectopic thyroid tissue are the strongest indicators and must be considered, and an assessment of volume could be useful.

The authors of this study believe problems with neck mobility could increase likelihood of sternotomy requirement. One sternotomy patient with a relatively small goitre was morbidly obese. No research could be found on these topics, so relationship of sternotomy to obesity or neck stiffness could be areas for future investigation.

It would be helpful if a consensus on the definition of retrosternal goitre could be reached, as currently the lack of standard definition makes studies difficult. The variety of definitions used across studies means there are large disagreements in results, and makes comparison of results and conclusions almost impossible. The 'clinical definition': 'a thyroid gland that on neck examination without being in hyperextension, has a portion that remains permanently retrosternal,' is the most inclusive definition, and is sensitive for determining presence of compressive symptoms and predicting difficult intubation. [8] Katlic's definition: $>50 \%$ 
of volume intrathoracic, is the most sensitive for predicting sternotomy, and an argument could be made that it is valuable to use this definition in certain research. [8] Use of other definitions is unlikely to be necessary and should be avoided. Some of the disagreement in the literature could also be down to surgical technique, experience, and attitude, as some surgeons are more reluctant or willing to use sternotomy. [12] only used sternotomy in 2 out of 355 patients $(0.6 \%)$ with retrosternal goitre, one of which was a primary retrosternal goitre, and the other a mediastinal recurrence of thyroid carcinoma. This shows that the reported rate of sternotomy in other studies may be higher than necessary. Small studies have reported good results from using less invasive methods than open thoracic surgery, such as VATS (video-assisted thoracoscopic surgery). [29, 30]

\section{Conclusion}

Volumetric analysis of retrosternal goitres has shown that there is a significant relationship between large volume and use of extracervical approaches for retrosternal thyroidectomy. An index of intrathoracic goitre volume to thoracic inlet diameter proved to be a more sensitive predictor than volume alone. Volumetric analysis would be useful as part of the preoperative radiological evaluation of retrosternal goitre patients, which should include assessment of goitre extension and position.

\section{Appendix}

Table A1. Descriptive statistics for volumetric analysis of retrosternal goitres for patients undergoing thyroidectomy by the cervical approach and sternotomy. Intrathoracic volume was the volume of the goitre inferior to the suprasternal notch. Volumetric index $=$ Intrathoracic volume $\div$ Thoracic inlet depth $\div 1000$.

\begin{tabular}{|c|c|c|c|c|c|c|c|c|c|}
\hline $\begin{array}{l}\text { Surgical } \\
\text { approach }\end{array}$ & Variable & $\begin{array}{l}\text { Number } \\
\text { patients }\end{array}$ & $\begin{array}{l}\text { Mean / } \\
\mathrm{mm}^{3}\end{array}$ & $\begin{array}{l}\text { Standard } \\
\text { Deviation }\end{array}$ & $\begin{array}{l}\text { Minimum } \\
\text { value / } \mathbf{m m}^{3}\end{array}$ & $\begin{array}{l}1^{\text {st }} \text { Quartile / } \\
\mathbf{m m}^{3}\end{array}$ & $\begin{array}{l}\text { Median / } \\
\mathrm{mm}^{3}\end{array}$ & $\begin{array}{l}3^{\text {rd }} \text { Quartile / } \\
\text { mm }^{3}\end{array}$ & $\begin{array}{l}\text { Maximum / } \\
\mathrm{mm}^{3}\end{array}$ \\
\hline \multirow{3}{*}{ Cervical } & Total Volume & 54 & 204785 & 106265 & 45507 & 126787 & 175775 & 301141 & 531821 \\
\hline & Intrathoracic Volume & 55 & 88063 & 78397 & 2938 & 29101 & 48732 & 151276 & 272141 \\
\hline & Volumetric Index & 54 & 1.651 & 1.547 & 0.053 & 0.546 & 0.915 & 2.511 & 6.545 \\
\hline \multirow[b]{2}{*}{ Sternotomy } & Total Volume & 9 & 263886 & 130112 & 91538 & 151457 & 211885 & 403945 & 435471 \\
\hline & Intrathoracic Volume & 9 & 196471 & 131013 & 33206 & 72471 & 167552 & 314915 & 409155 \\
\hline
\end{tabular}

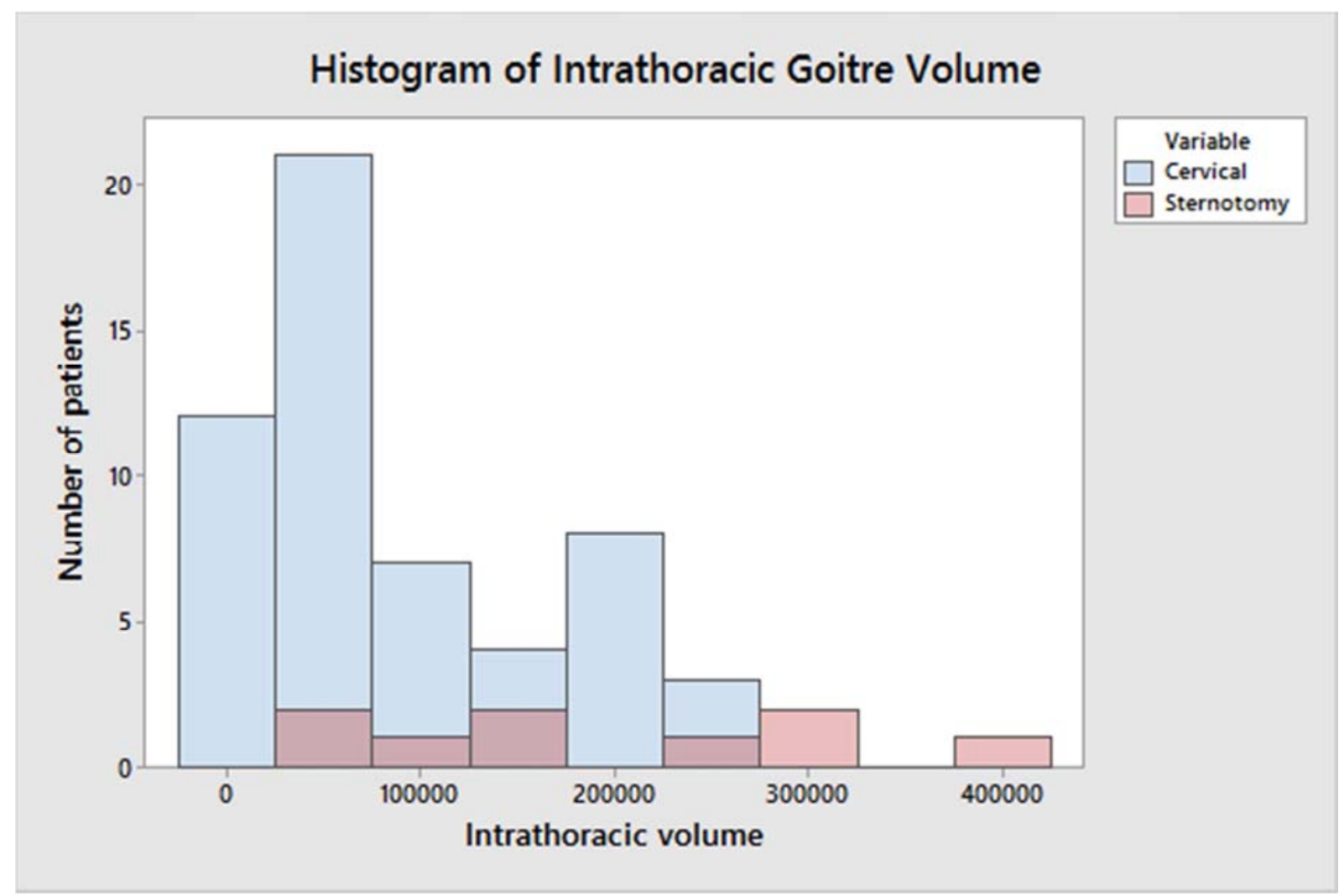

Figure A1. Histogram showing distribution of intrathoracic goitre volume in retrosternal thyroidectomy patients. Blue bars indicate patients who required only a cervical incision to remove their goitre. Red bars indicate patients who required a thoracic approach. Intrathoracic volume was the volume inferior to the level of the suprasternal notch. Patients requiring sternotomy generally had larger intrathoracic goitre volume. 


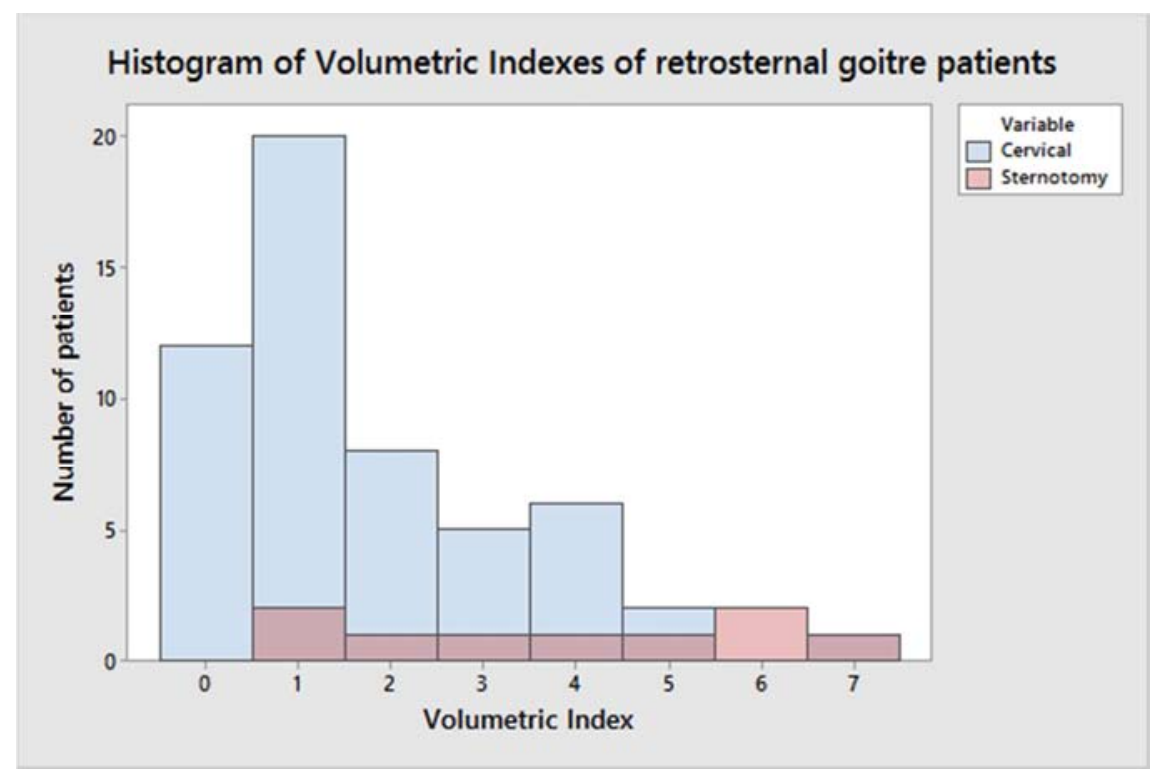

Figure A2. Histogram showing distribution of volumetric indexes for retrosternal thyroidectomy patients. Blue bares indicate patients who required only a cervical incision to removes their goitre. Red bars indicate patients who required a thoracic approach. Volumetric Index $=$ Intrathoracic volume $\div$ Thoracic inlet depth $\div 1000$. Patients requiring sternotomy had larger volumetric indexes.

\section{References}

[1] Lahey, F. H. \& Swinton, N. W. 1934. Intrathoracic goiter. Surgery Gynecology \& Obstetrics, 59, 627-637.

[2] Netterville, J. L., Coleman, S. C., Smith, J. C., Smith, M. M., Day, T. A. \& Burkey, B. B. 1998. Management of substernal goiter. Laryngoscope, 108, 1611-1617.

[3] Bizakis, J., Karatzanis, A., Hajiioannou, J., Bourolias, C., Maganas, E., Spanakis, E., Bizaki, A. \& Velegrakis, G. 2008. Diagnosis and management of substernal goiter at the University of Crete. Surgery Today, 38, 99-103.

[4] Coskun, A., Yildirim, M. \& Erkan, N. 2014. Substernal Goiter: When is a Sternotomy Required? International Surgery, 99, 419-425.

[5] Cohen, J. R. 2009. Substernal Goiters and Sternotomy. Laryngoscope, 119, 683-688. Coskun, A., Yildirim, M. \& Erkan, N. 2014. Substernal Goiter: When is a Sternotomy Required? International Surgery, 99, 419-425.

[6] Testini, M., Gurrado, A., Avenia, N., Bellantone, R., Biondi, A., Brazzarola, P., Calzolari, F., Cavallaro, G., De Toma, G., Guida, P., Lissidini, G., Loizzi, M., Lombardi, C. P., Piccinni, G., Portincasa, P., Rosato, L., Sartori, N., Zugni, C. \& Basile, F. 2011. Does Mediastinal Extension of the Goiter Increase Morbidity of Total Thyroidectomy? A Multicenter Study of 19,662 Patients. Annals of Surgical Oncology, 18, 2251-2259.

[7] White, M. L., Doherty, G. M. \& Gauger, P. G. 2008. Evidence-based surgical management of substernal goiter. World Journal of Surgery, 32, 1285-1300.

[8] Rios, A., Rodriguez, J. M., Balsalobre, M. D., Tebar, F. J. \& Parrilla, P. 2010. The value of various definitions of intrathoracic goiter for predicting intra-operative and postoperative complications. Surgery, 147, 233-238.

[9] El Oueriachi, F., El Hammoumi, M. M., Arsalane, A., Slaoui,
O. \& Kabiri, E. 2014. Primary mediastinal goiters. Springerplus, 3,5 .

[10] Rugiu, M. G. \& Piemonte, M. 2009. Surgical approach to retrosternal goitre: do we still need sternotomy? Acta Otorhinolaryngologica Italica, 29, 331-338.

[11] Chen, A. Y., Bernet, V. J., Carty, S. E., Davies, T. F., Ganly, I., Inabnet, W. B. \& Shaha, A. R. 2014. American Thyroid Association Statement on Optimal Surgical Management of Goiter. Thyroid, 24, 181-189.

[12] Raffaelli, M., De Crea, C., Ronti, S., Bellantone, R. \& Lombardi, C. P. 2011. SUBSTERNAL GOITERS: INCIDENCE, SURGICAL APPROACH, AND COMPLICATIONS IN A TERTIARY CARE REFERRAL CENTER. Head and Neck-Journal for the Sciences and Specialties of the Head and Neck, 33, 1420-1425.

[13] Cichon, S., Anielski, R., Konturek, A., Baczynski, M., Cichon, W. \& Orlicki, P. 2008. Surgical management of mediastinal goiter: risk factors for sternotomy. Langenbecks Archives of Surgery, 393, 751-757.

[14] Khan, M. N., Goljo, E., Owen, R., Park, R. C. W., Yao, M. \& Miles, B. A. 2016. Retrosternal Goiter: 30-Day Morbidity and Mortality in the Transcervical and Transthoracic Approaches. Otolaryngology-Head and Neck Surgery, 155, 568-574.

[15] Rolighed, L., Ronning, H. \& Christiansen, P. 2015. Sternotomy for substernal goiter: retrospective study of 52 operations. Langenbecks Archives of Surgery, 400, 301-306.

[16] Casella, C., Pata, G., Cappelli, C. \& Salerni, B. 2010. PREOPERATIVE PREDICTORS OF STERNOTOMY NEED IN MEDIASTINAL GOITER MANAGEMENT. Head and Neck-Journal for the Sciences and Specialties of the Head and Neck, 32, 1131-1135.

[17] Malvemyr, P., Liljeberg, N., Hellstrom, M. \& Muth, A. 2015. Computed tomography for preoperative evaluation of need for sternotomy in surgery for retrosternal goitre. Langenbecks Archives of Surgery, 400, 293-299. 
[18] Nankee, L., Chen, H., Schneider, D. F., Sippel, R. S. \& Elfenbein, D. M. 2015. Substernal goiter: when is a sternotomy required? Journal of Surgical Research, 199, 121125 .

[19] Qureishi, A., Garas, G., Tolley, N., Palazzo, F., Athanasiou, T. \& Zacharakis, E. 2013. Can pre-operative computed tomography predict the need for a thoracic approach for removal of retrosternal goitre? International Journal of Surgery, 11, 203-208.

[20] Mckenzie, G. A. G. \& Rook, W. 2014. Is it possible to predict the need for sternotomy in patients undergoing thyroidectomy with retrosternal extension? Interactive Cardiovascular and Thoracic Surgery, 19, 139-143.

[21] Riffat, F., Del Pero, M. M., Fish, B. \& Jani, P. 2013. Radiologically Predicting When a Sternotomy May Be Required in the Management of Retrosternal Goiters. Annals of Otology Rhinology and Laryngology, 122, 15-19.

[22] Sari, S., Erbil, Y., Ersoz, F., Saricam, G., Salmaslioglu, A., Issever, H. \& Ozarmagan, S. 2012. Predictive Value of Thyroid Tissue Density in Determining the Patients on Whom Sternotomy Should be Performed. Journal of Surgical Research, 174, 312-318.

[23] Mercante, G., Gabrielli, E., Pedroni, C., Formisano, D., Bertolini, L., Nicoli, F., Valcavi, R. \& Barbieri, V. 2011. CT CROSS-SECTIONAL IMAGING CLASSIFICATION SYSTEM FOR SUBSTERNAL GOITER BASED ON RISK FACTORS FOR AN EXTRACERVICAL SURGICAL APPROACH. Head and Neck-Journal for the Sciences and Specialties of the Head and Neck, 33, 792-799.
[24] Latini, F., Larsson, E.-M. \& Ryttlefors, M. 2015. Rapid and Accurate MRI Segmentation of Peritumoral Brain Edema in Meningiomas. Clinical Neuroradiology, 1-8.

[25] Folio, L. R., Sandouk, A., Huang, J. X., Solomon, J. M. \& Apolo, A. B. 2013. Consistency and Efficiency of CT Analysis of Metastatic Disease: Semiautomated Lesion Management Application Within a PACS. American Journal of Roentgenology, 201, 618-625.

[26] Katlic, M. R., Wang, C. \& Grillo, H. C. 1985. SUBSTERNAL GOITER. Annals of Thoracic Surgery, 39, 391-399.

[27] Chang, C. Y., Hong, Y. C., Chung, P. C. \& Tseng, C. H. 2011. A Neural Network for Thyroid Segmentation and Volume Estimation in CT Images. Ieee Computational Intelligence Magazine, 6, 43-55.

[28] Chen, A., Niermann, K. J., Deeley, M. A. \& Dawant, B. M. 2012. Evaluation of multiple- atlas-based strategies for segmentation of the thyroid gland in head and neck CT images for IMRT. Physics in Medicine and Biology, 57, 93-111.

[29] Bhargav, P. R., Amar, V., Mahilvayganan, S. \& Nanganandadevi, V. 2016. Feasibility of thoracoscopic approach for retrosternal goitre (posterior mediastinal goitre): Personal experiences of 11 cases. Journal of Minimal Access Surgery, 12, 240-244.

[30] Gupta, P., Lau, K. K. W., Rizvi, I., Rathinam, S. \& Waller, D. A. 2014. Video assisted thoracoscopic thyroidectomy for retrosternal goitre. Annals of the Royal College of Surgeons of England, 96, 606-608. 